\title{
Emerging treatment modalities for advanced pancreatic neuroendocrine tumors
}

\author{
Alessandro Sindoni, ${ }^{1}$ Fausto Famà, ${ }^{2}$ Maria Gioffre-Florio, ${ }^{2}$ Sergio Baldari ${ }^{1}$ \\ ${ }^{1}$ Department of Biomedical and Dental Sciences and of Morphological and Functional Images, ${ }^{2}$ Department of Human \\ Pathology, University Hospital of Messina, Messina, Italy
}

Dear Sir,

It was with great interest that we read the article titled "Neoadjuvant therapy for advanced pancreatic neuroendocrine tumors: an emerging treatment modality?" by I. Perysinakis et al published in the first issue of 2016 of the Journal. ${ }^{1}$ The authors review the recent literature concerning advanced pancreatic neuroendocrine tumors (pNETs) and highlight the fact that there is increasing evidence supporting the application of neoadjuvant protocols in this set of patients aiming at tumor downsizing to render curative surgery feasible.

Complete surgical resection is the only potentially curative treatment of localized pNETs, ${ }^{1}$ but a significant proportion of patients affected by pNETs have inoperable locally advanced tumors and/or metastatic disease, which make the surgical approach infeasible. ${ }^{2,3}$ In this setting, the proposed goal is to

Key words: Pancreatic neuroendocrine tumors, Peptide receptor radionuclide therapy, ${ }^{177}$ Lu-DOTATATE

Address for correspondence:

Alessandro Sindoni, M.D. Dipartimento di Scienze Biomediche, Odontoiatriche e delle Immagini Morfologiche e Funzionali,

1 Via Consolare Valeria, 98125 Messina, Italy;

Tel.: +39090 2212240, Fax: +39090 2213192,

E-mail: alessandrosindoni@alice.it

Received: 28-08-2016, Accepted: 10-09-2016 perform neoadjuvant therapy, followed by surgery in responders. ${ }^{1}$ Several preoperative approaches have been reported in the medical literature, these options including chemotherapy, radiotherapy, peptide receptor radionuclide therapy (PRRT), biological drugs or combinations of treatments.

PRRT is a useful therapeutic approach for the treatment of somatostatin receptor positive NETs and usually follows failed first-line medical therapy. ${ }^{90} \mathrm{Y}$ and/or ${ }^{177}$ Lu-labeled somatostatin analogues, which are increasingly used in pNETs patients, are also characterized by lower nephrotoxicity. The phase III NETTER-1 trial has provided evidence of significant benefit in progression free-survival and overall survival in patients with advanced midgut NETs treated with ${ }^{177} \mathrm{Lu}$-DOTATATE, and its safety profile was found to be favorable in terms of hematological, renal and hepatic toxicities. ${ }^{4}$

The use of antiproliferative therapies is as yet challenging and varies on the basis of the disease burden, relative functional status and growth rate and on the possible eventual development of comorbidities. Recommendations for preferential use of targeted drugs, chemotherapy or PRRT as first-line therapy have also recently been suggested. ${ }^{5}$ However, the potential of augmented toxicity, such as after prior chemotherapy or targeted therapy, calls for adequate surveillance and can justify an earlier use of PRRT in selected patients. ${ }^{5}$ The advantages offered by PRRT are not limited to therapeutic purposes but also serve as diagnostic tools. In fact, after administra- 
tion of PRRT, patients usually undergo post-therapy whole-body scans (PTWBS) to evaluate radiopharmaceutical biodistribution. As demonstrated in our previous study, ${ }^{6}$ PTWBS imaging can reveal more lesions than pretherapy imaging; thus, PTWBS can better delineate the extent of disease than standard diagnostic procedures, allowing the visualization of additional lesions and permitting an accurate staging of disease, thus enabling optimization of the clinical management of patients. Taking into account all these advantages we consider, as do I. Perysinakis et al, ${ }^{1}$ that expert consensus about locally advanced pNETs is needed to assess the benefits of neoadjuvant treatments and define optimal treatment schedules including PRRT.

\section{CONFLICT OF INTEREST STATEMENT}

The authors declare that there is no conflict of interest and that they received no specific funding for this article.

\section{REFERENCES}

1. Perysinakis I, Aggeli C, Kaltsas G, Zografos GN, 2016 Neoadjuvant therapy for advanced pancreatic neuroendocrine tumors: an emerging treatment modality? Hormones (Athens) 15: 15-22.

2. Frilling A, Clift AK, 2015 Therapeutic strategies for neuroendocrine liver metastases. Cancer 121: 1172-1186.

3. Kaltsas GA, Besser GM, Grossman AB, 2004 The diagnosis and medical management of advanced neuroendocrine tumors. Endocr Rev 25: 458-511.

4. Strosberg J, Wolin E, Chasen B, et al, 2016 NETTER-1 phase III in patients with midgut neuroendocrine tumors treated with 177lu-dotatate: Efficacy and safety results. J Nucl Med 57: Suppl 2: 629.

5. Pavel M, O'Toole D, Costa F, et al; Vienna Consensus Conference Participants, 2016 ENETS consensus guidelines update for the management of distant metastatic disease of intestinal, pancreatic, bronchial neuroendocrine neoplasms (NEN) and NEN of unknown primary site. Neuroendocrinology 103: 172-185.

6. Minutoli F, Herberg A, Sindoni A, Cardile D, Cucinotta M, Baldari S, 2013 Peptide receptor imaging in neuroendocrine tumors: comparison between diagnostic scintigraphy and post-therapy whole-body scan. Ann Nucl Med 27: 654-660. 